\title{
BMJ Open The effects of a mindfulness-based lifestyle programme for adults with Parkinson's disease: protocol for a mixed methods, randomised two-group control study
}

\author{
Jenny Advocat, ${ }^{1}$ Grant Russell, ${ }^{1,2}$ Joanne Enticott, ${ }^{1}$ Craig Hassed, ${ }^{3}$ \\ Jennifer Hester, ${ }^{2}$ Brooke Vandenberg ${ }^{1}$
}

To cite: Advocat J, Russell G, Enticott J, et al. The effects of a mindfulness-based lifestyle programme for adults with Parkinson's disease: protocol for a mixed methods, randomised twogroup control study. BMJ Open 2013;3:e003326. doi:10.1136/bmjopen-2013003326

- Prepublication history for this paper is available online. To view these files please visit the journal online (http://dx.doi.org/10.1136/ bmjopen-2013-003326).

Received 31 May 2013 Accepted 13 September 2013

For numbered affiliations see end of article.

Correspondence to Dr Joanne Enticott; joanne. enticott@monash.edu

\section{ABSTRACT}

Introduction: Parkinson's disease (PD) is the second most common neurodegenerative disorder in developed countries. There is an increasing interest in the use of mindfulness-related interventions in the management of patients with a chronic disease. In addition, interventions that promote personal control, stress-management and other lifestyle factors, such as diet and exercise, assist in reducing disability and improving quality of life in people with chronic illnesses. There has been little research in this area for people with PD.

Methods: A prospective mixed-method randomised clinical trial involving community living adults with PD aged $<76$ years and with moderate disease severity (Hoehn and Yahr stage 2) PD. Participants will be randomised into the ESSENCE 6-week programme or a matched wait list control group. ESSENCE is a multifaceted, healthy lifestyle and mindfulness programme designed to improve quality of life. We aim to determine whether participation in a mindfulness and lifestyle programme could improve PD-related function and explore self-management related experiences and changing attitudes towards self-management. The outcome measures will include 5 self-administered questionnaires: PD function and well-being questionnaire (PDQ39), Health Behaviours, Mental health, Multidimensional locus of control, and Freiburg mindfulness inventory. An embedded qualitative protocol will include in-depth interviews with 12 participants before and after participation in the 6-week programme and a researcher will observe the programme and take notes.

Analysis: Repeated measures of Analysis of Variance (ANOVA) will examine the outcome measures for any significant effects from the group allocation, age, sex, adherence score and attendance. Qualitative data will be analysed thematically. We will outline the benefits of, and barriers to, the uptake of the intervention.

Ethics: This protocol has received ethics approval from the Monash University Human Research Ethics Committee project number CF11/2662-2011001553.

Dissemination: This is the first research of its kind in Australia involving a comprehensive, lifestyle-based programme for people with PD and has the potential to involve a broader range of providers than standard care. The findings will be disseminated through peer reviewed journals, primary care conferences in Australia as well as abroad and through the Parkinson's community.

Registration details: Australian New Zealand Clinical Trials Registry (ANZCTR) ACTRN12612000440820.

\section{INTRODUCTION}

Parkinson's disease (PD) is the second commonest neurodegenerative disorder in developed countries. PD affects $1 \%$ of people over 60 years of age and $4 \%$ of those aged over $80 .^{1}$ As in other western nations, Australia's changing population demographics will generate a threefold increase in the incidence of the condition by $2033 .^{2}$ Despite high-quality evidence demonstrating pharmacological benefits in managing symptoms and delaying disease progression, ${ }^{3}$ there is still no known cure for PD. ${ }^{4}$

Evidence is accumulating that lifestyle interventions may reduce disability in people living with neurodegenerative disorders similar to that of PD. ${ }^{5}$ Mindfulness, principally understood as the ability to train attention, has slowed disease progression and improved quality of life in patients with heart disease and cancer. ${ }^{6} 7$ Programmes using combinations of healthy eating, regular exercise and stress management have improved outcomes in people living with cancer, ${ }^{8}$ fibromyalgia, ${ }^{9}$ rheumatoid arthritis ${ }^{10}$ and chronic low back pain. ${ }^{11}$ Meta-analyses on lifestyle interventions have suggested improvements in depression, anxiety and psychological distress in people with somatic diseases. ${ }^{12}$ Clear benefits from lifestyle interventions in 
patients with various chronic diseases are now evident, ${ }^{6}{ }^{7}$ 13-16 making it logical to also examine lifestyle factors in people living with PD.

The ESSENCE Programme is an innovative, holistic lifestyle and mindfulness programme that aims to improve quality of life and self-management of stress and/or chronic disease. ${ }^{17}$ ESSENCE is an acronym for: Education, Stress management, Spirituality, Exercise, Nutrition, Connectedness and Environment. ESSENCE has already assisted people with multiple sclerosis ${ }^{18}$ and mental health in students. ${ }^{19-21}$ This study aims to investigate if people living with $\mathrm{PD}$, who participate in the ESSENCE programme, can experience an improvement in PD-related function and well-being. In addition, we will seek to ascertain the experiences of participants in the programme, particularly in relation to their ability to self-manage the effects and symptoms of PD.

\section{METHODS AND ANALYSIS \\ Study design}

This study is a prospective mixed-method randomised clinical trial incorporating a before and after design with a waitlist control. The study is to operate over 12 months in 2012/2013.

\section{Setting}

The ESSENCE programmes will be delivered at two venues, which will be located in two different inner urban suburbs of Melbourne, Victoria. These suburbs are separated by the central business district and are approximately $20 \mathrm{~km}$ apart. The venue at each location will be an easily accessible, local community centre. Both venues will be located close to an existing large PD support group.

\section{Formation of advisory group}

An expert advisory group will be formed for the purpose of the study to advise on recruitment, link clinicians and researchers and provide comment to maintain relevance of the research. The advisory group will meet three times during the 12-month project period. Members of the advisory group will consist of health professionals with an interest in PD, two people living with PD and the research team. The health professionals include two neurologists, two health psychologists, one occupational therapist, one representative from the state support and funding body Parkinson's Victoria (PV) and another from the state PD registry (Victorian Parkinson's registry). Apart from contributions to the advisory committee, the funder will have no role in decisions and procedures concerning data collection, analysis, interpretation and academic dissemination.

\section{Participants}

The 'test' group are to be participants attending the first 6-week ESSENCE programme at each location. The controls will be those in each waitlist group. All the control participants will be invited to attend a second ESSENCE programme held at each site, which starts after the test groups have completed the programme. An initial study eligibility criterion for participants was $18-70$ years of age. This was reviewed by the advisory group and changed to include participants up to 75 years of age in order to maximise recruitment reach and improve study power.

The eligibility criteria are

- Between the ages of 18 and 75

- Fluent in English

- Able to attend at least four of six sessions of a ESSENCE programme

- The community living adults with disability congruent with Hoehn and Yahr stage two PD. ${ }^{22}$ This staging, for the purposes of this study, is to be determined by screening participants using the two below questions. To be eligible, participants must answer 'yes' to both questions:

- 'Do you have problems of shaking (tremor), stiffness or difficulty with movements on both sides of your body?'

- 'Most of the time you can you walk straight and stand up without assistance? (ie, not severe)'

\section{Recruitment}

Volunteer participants will be recruited from the PD community residing in metropolitan Melbourne. Target participants will initially be invited to participate through a combination of written invitations from Parkinson's Victoria to those listed on the Parkinson's registry, advertisements in Parkinson's Victoria publications (written and electronic) and invitations disseminated to clinicians dealing with patients with PD. As highlighted from a number of previous studies within the Parkinson's community, recruitment within this population is significantly slower than most other chronic disease populations resulting in low study participant numbers. ${ }^{23}$ In order to address this potential barrier to our study, a recruitment officer will be employed. The recruitment officer will assist the advisory board in developing additional recruitment strategies.

Such additional strategies to be considered mirror those addressed by the Michael J. Fox Foundation. ${ }^{23}$ These will include the development of promotional study materials including posters, flyers delivered to the Melbourne-based community and health services, engaging with Parkinson's support groups, presenting at major Parkinson's Victoria events including the Parkinson's 'Walk in the Park' and Young at Parkinson's annual meeting, utilising existing recruitment registries such as the Michael. Fox Foundation clinical trial finder registry.

Recruitment is expected to take 6 months and will end 1-week prior to the start of the test programmes.

\section{Sample size}

We calculated the sample size to detect a medium-to-large change in the primary outcome PD questionnaire- 
summary index (PDQ39-SI). ${ }^{24}$ Calculations were based on published data from participants with Hoehn and Yahr stage two PD (PDQ39-SI mean 31.6, SD 17.00). ${ }^{25}$ Hence, an improvement of 10 points on the PDQ39-SI would be detectable with 0.8 power with between two samples having 30 participant each (one-sided t test).

The recruitment target is set at 80 . Although the study requires 60 participants, a drop-out rate of $25 \%$ is anticipated due to similarly high drop-out rates experienced by other studies involving participants with PD. ${ }^{23}$ Sample sizes of approximately 30 participants in each of the test and control groups will produce sufficient power to examine the quantitative hypotheses. ${ }^{26}$ Other mindfulness programmes have found significant effects with similar sample sizes. ${ }^{27-29}$ Medium to large effect sizes are to be investigated. ${ }^{26}$

\section{Randomisation}

Allocation to a test or control group will be by random number generation. The permuted block method using blocks of four ${ }^{30}{ }^{31}$ has been chosen because it will assist with creating balanced numbers within groups. Prior to the creation of the randomisation allocation code, each participant will elect a programme location, either location A or B. Two randomisation codes will be created, one for each location. Both codes will be created 1-week prior to the start of the ESSENCE programmes for test participant. The codes will be kept at the central administration site which is located 'off-site.'

Participant names will be allocated to either the test or control group using the randomised allocation code for each location. The recruitment officer remains unaware of the code until participants have been allocated into a group. The recruitment officer will notify the participant of the start date for their allocated group immediately after the key is created. Participants (and the recruitment officer) are unable to change any allocated group. Participants who are unable to attend their allocated session will be thanked for their willingness to participate in research and then disqualified from the study.

\section{Intervention}

Participation in the 6-week ESSENCE programme ${ }^{17}$ will involve participants attending a $2 \mathrm{~h}$ group session, once a week for a total period of 6 weeks.

The ESSENCE programme is designed to introduce key mindfulness and lifestyle elements to participants (figure 1). The programme introduces simple strategies that can help participants to live better with chronic disease. Participants will be encouraged to apply aspects of the ESSENCE model that are most relevant to them in their own lives. Mindfulness training and group support, provided by the regular, weekly sessions involving other people at a similar stage of progression of PD, are part of the programme to provide benefit in their own right as well as to facilitate healthy lifestyle change.
The programme will be facilitated by the creator of the ESSENCE model and General Practitioner (CH) whose role is to run the programme and not to intervene in the medical management of the participants' PD. Any ongoing medical or mental health problems that might be identified throughout the programme are to be referred back to the participants' treating doctors. The programme facilitator is to be blinded to all participant data.

\section{Embedded qualitative protocol}

An embedded qualitative protocol will explore the experiences of participating in the ESSENCE programme, including an aim to uncover the effect of the programme on participants' beliefs about PD and its management, how attitudes towards health efficacy may have evolved, aspects of the intervention which were most valuable and meaningful and level of participant engagement. This part of the study requires a maximum variation sample (by gender and age) of between 10 and 12 test participants to participate in up to three interviews.

All participants in the test group will be contacted and asked to participate in this substudy. Participants will be selected and contacted by the qualitative researcher. These participants will participate in at least two interviews: one prior to the start of the programme and a final interview within the month after the completion of the 6-week programme. Interviews will be scheduled at a time and place most convenient to the participant.

\section{Outcome measures}

\section{Quantitative data}

The primary outcome is the change in function and well-being associated with PD experienced by the test group at the conclusion of the ESSENCE programme as compared with the waitlist controls. Secondary outcomes will be changes in (A) health behaviours, (B) mental health and (C) locus of control. These outcome variables will be measured by participants completing five validated self-administered questionnaires:

- Function and well-being associated with PD $\left(\right.$ PDQ39) ${ }^{24}$

- Health Behaviours Questionnaire ${ }^{32}$

- Mental health (DASS-21) ${ }^{33}$

- Multidimensional locus of control ${ }^{34}$

- Freiburg mindfulness inventory ${ }^{35}$

Test and control participants will complete the study questions at three time points: baseline, week 7 and again at 6 months (see table 1 ).

Baseline questionnaires will be mailed to all test and control participants with a cover letter requesting that questionnaires are completed and returned within 1 week. A self-addressed, postage paid, return envelope and email or telephone reminders will be included to encourage prompt returns. The cover letter will explain that if a participant requires any assistance to complete the questionnaires then they can call (or email) the 


\section{Education}

- Knowledge about the condition and its management

- Information on the effects of lifestyle factors on health including reducing, or eliminating harmful behaviours and increasing health promoting behaviours

\section{Stress management}

- Promoting group-support, meditations and greater emotional intelligence

Spirituality

- Increasing individual's ability to explore personal 'meaning' and/or 'purpose' in life

Exercise

- Promoting increased physical activity for reduced risk of chronic disease and improved mental health outcomes, such as self-esteem, depression and anxiety

\section{Nutrition}

- Encouraging health nutrition though the provision of information on the benefits of micronutrients in food and outcomes of a diabetogenic diet

- Promoting simple strategies for improving health food choices

Connectedness

- Information about the importance of professional and emotional support in the self-management of chronic disease

\section{Environment}

- Raising awareness of the importance of reducing exposure to harmful chemicals

- Promoting increased sun exposure (at safe levels)

* Hassed C. The ESSENCE of health care. Aust Fam Physician 2005;34(11):957-960

Figure 1 The ESSENCE acronym.

researchers for assistance. Researcher contact details will be indicated in the cover letter. Participants will be emailed a link to an electronic instructional video, detailing each component of the questionnaire. ${ }^{36}$ Any test group participants presenting to the first session without having completed the baseline questionnaires, will be required to complete them before the session starts.

Week 7 questionnaires will be distributed to the test participants after the final session. If a participant is absent then the questionnaires will be posted, and the participant contacted by phone and email to encourage returns. Control participants will receive the week 7 questionnaires in the mail, again with a return selfaddressed envelope. The cover letter will explain that repeating these questionnaires is important for the study. Follow-up phone calls and emails will begin at week 8, if necessary, to encourage returns. Questionnaires will be deemed ineligible if they are not returned by the end of week 10 .

\section{Other quantitative data}

Participant attendance will be monitored. Each test group session will be attended by a researcher in addition to the course facilitator. The researcher will be responsible for completing the attendance role. If a participant cannot attend four or more sessions, then this will be recorded and considered as a factor in the analysis.

Participant adherence will be measured. This will be done by test participants self-rating their practice of the ESSENCE exercises using a modified medical outcomes study. ${ }^{37}$ Figure 2 shows our centre-developed short questionnaire, which will quantify the level of adherence to the programme. This adherence measure will be administered three times to test participants and only once to the controls (table 1). Test participants will be required to complete the questionnaire prior to leaving the fourth and final sessions. All participants will be mailed the questionnaire along with the other study questionnaires in weeks 26-32 (at the 6-month study time point). 


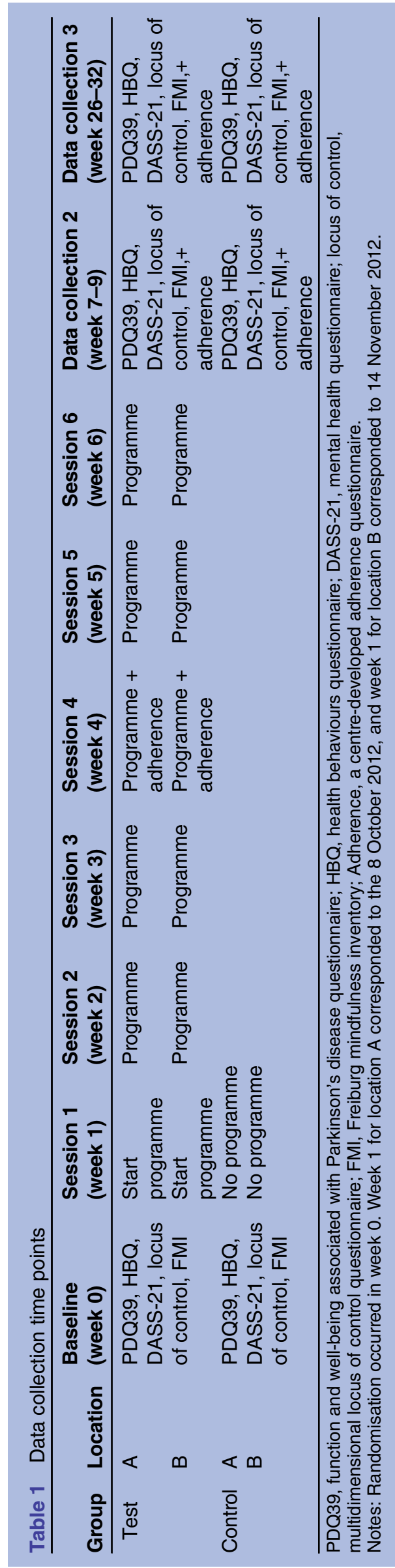

Qualitative data

Data collection will involve semistructured, in-depth interviews as well as participant observation of the programme. Semistructured interviews will elicit participants' health beliefs and illness narratives and will focus on the central questions of this study. Interviews will be digitally recorded and transcribed verbatim. The interviewer will be an experienced qualitative health researcher.

Observation will involve a research assistant attending all sessions of the early intervention groups in order to inform the postprogramme interview structure. Notes will be taken during and directly following observation and these notes will contribute to the data set.

\section{Data analysis}

Quantitative data

Data will be entered in a spreadsheet (eg, Microsoft Excel) by one research assistant. The quality of data entry will be checked by a second research assistant performing double data entry of $10 \%$ of the data. Nine to eight per cent agreement between the two entered datasets will be the cut-off criteria for acceptable quality. If there is less than $98 \%$ agreement then the entire dataset will be re-entered and mistakes corrected using a continuous sampling plan. ${ }^{38}$

The patient demographic information, such as age and gender, will be examined in relation to the outcome measures. In addition, adherence score and group allocation will be recorded as factors. Data will be examined for normality distributions and if non-normality exists then Kruskal-Wallis analysis will be undertaken to examine the data. Repeated measures Analysis of Variance (ANOVA) will examine the data for any significant effects from multiple factors including age, sex, group allocation, adherence score and attendance. In addition, outcomes of the programme will be correlated with participant scores for high medium and low adherence using Pearson's regression. ${ }^{39}$

\section{Qualitative data}

Qualitative data analysis will involve investigating the interview transcripts, interview notes and observational field notes taken by the researchers. The analysis will follow typical qualitative techniques including iterative analysis $^{40}$ and immersion/crystallisation. ${ }^{41}$ Data will be prepared for analysis by entering transcripts, field notes and recordings in the NVivo software programme. The analysis will begin with the first interview and will form an iterative process of exploring new and unanticipated research questions and developing and testing various ways of interpreting the data. ${ }^{40}$ Searches will be made for disconfirming cases, and continual conceptual refinement will be made throughout the analysis. ${ }^{42}$

\section{ETHICS AND DISSEMINATION}

The expert advisory group will be an important conduit for the initial dissemination of findings. The group will 
How often was each of the following statem ents true for you during the past 2 weeks?

1. I had a hard time doing what the ESSENCE program suggested I do...

2. I followed the ESSENCE program suggestions exactly...

3. I was unable to do what was necessary to follow the recomm endations in the ESSENCE program...

4. I found it easy to do the things the ESSENCE program suggested I do...

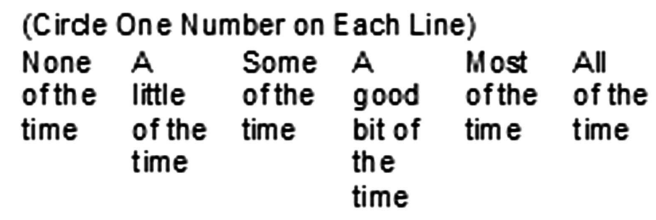

\begin{tabular}{llllll}
\hline 1 & 2 & 3 & 4 & 5 & 6
\end{tabular}

1

1

2
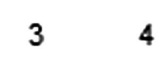

5

6

1

2

3

4

56

1

2

3

4

5

6

5. Generally speaking, how often during the past 2 weeks were you able to do what was recommended in the ESSENCE program?

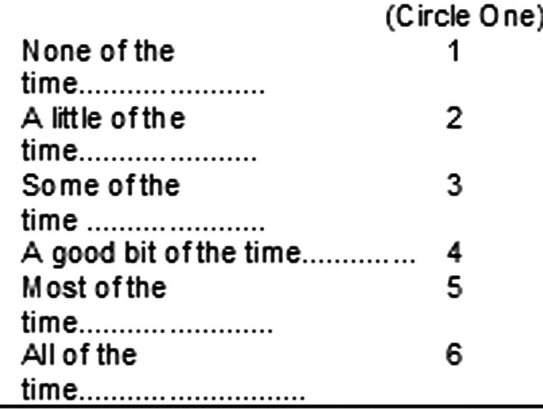

Figure 2 Intervention adherence from participants rate their practice of the ESSENCE exercises using this modified medical outcomes study questionnaire. It asks participants to rate the type, frequency and duration of 'ESSENCE and stress management techniques'used in the previous 3 weeks. Adherences scores will be calculated by adding the numeric scores circled by the participant in the nine item questionnaire, and then dividing the cumulative score by 54 (ie,divide by the highest possible cumulative score). A total score of 1 will indicate $100 \%$ adherence to the recommended if estyle change programme, and $\mathrm{O}$ indicates no adherence.

also provide advice on additional dissemination methods to make the greatest impact within the study field. The study findings will be made accessible to people with PD within Victoria, and potentially throughout Australia, through project updates and outputs placed on websites and newsletters of the advisory group forums and funding body Parkinson's Victoria. With the academic expertise of the investigators, study findings will also be disseminated through academic outlets including journal publications, national and international conferences and peer-reviewed forums.

A potential ethical issue of consideration in this study may arise if any study participant is identified as having significant depression as indicated by the DASS21 ${ }^{33}$ (collected at baseline, week 7 and again at 6 months). The MUHREC was consulted about this possible scenario.
They advised that such patients could participate in the programme providing that a clinical researcher associated with the project confidentially discusses the abnormal depression scores with the participant and recommend that they see their general practitioner. This minimal follow-up was deemed as appropriate because the programme intervention is unlikely to cause harm particularly in light of the participants only practicing mindfulness-based strategies and making lifestyle changes according to their own motivation and choice.

The study protocol was approved by the MUHREC Project number CF11/2662-2011001553. Data will be securely stored for 5 years in accordance with MUHREC guidelines. The trial was registered with the Australian and New Zealand Clinical Trial Register (ACTRN12612000440820) on 18/04/ 2012. 


\section{DISCUSSION}

The last decade has seen an explosion in the trialling of mindfulness-based interventions in the management of chronic disease. $^{27}$ 43-46 Our mixed-methods, pragmatic, study differs from many of these interventions by its use of a community sample and its incorporation of methods designed to capture the comprehensive and holistic nature of the ESSENCE programme.

Unlike many randomised trials we will seek an understanding of participant experience in the intervention. We seek representation from people living with PD and those responsible for organising care. Our expert advisory panel has already provided invaluable advice particularly in preparing for recruitment and delivery of the intervention. They encouraged our approaches to carer participation, venue accessibility and event timing.

Non-obtrusive observation by a researcher will give us an additional perspective of participation. A degree of 'ethnographic distance ${ }^{47}$ can allow us to consider the sorts of things that are meaningful to those studied. Specifically: What do participants stop and watch? What do they talk and gossip about? What produces strong emotional responses for them? ${ }^{47}$ Such reflexive distance is useful for qualitative analysis but in this study we will supplement this with a close relationship with our expert advisory group.

Our pragmatic study will be an important first step in exploring the impact of a new approach to the selfmanagement of this complex chronic condition, and, in parallel, illuminate some of the complexities faced by those living with PD.

\section{Author affiliations \\ ${ }^{1}$ Southern Academic Primary Care Research Unit, Dandenong, Victoria, Australia \\ ${ }^{2}$ School of Primary Health Care, Monash University, Notting Hill, Victoria, Australia \\ ${ }^{3}$ Department of General Practice, Monash University, Notting Hill, Victoria, Australia}

Contributors GR was the chief investigator, responsible for the overall development for a quality and ethical study protocol. JA was involved in the initial conception of the study in collaboration with $\mathrm{GR}$ and $\mathrm{CH}$. GR and $\mathrm{CH}$ are the qualitative expertise, questionnaire design/identification and liaison with stakeholders. JE was the quantitative methodology expertise, design refinement and liaison with stakeholders and prepared the manuscript with all authors providing substantial contributions. $\mathrm{CH}$ designed and adapted the study intervention. JH was the qualitative methodology expert, along with design refinement and recruitment advice. BV was involved in the recruitment advice, liaison with stakeholders and manuscript editing.

Funding This work was supported through a research grant by Parkinson's Victoria

Competing interests None.

Ethics approval Monash University Human Research Ethics Committee (MUHREC) approval no. CF11/2662-2011001553.

Provenance and peer review Not commissioned; internally peer reviewed.

Open Access This is an Open Access article distributed in accordance with the Creative Commons Attribution Non Commercial (CC BY-NC 3.0) license, which permits others to distribute, remix, adapt, build upon this work noncommercially, and license their derivative works on different terms, provided the original work is properly cited and the use is non-commercial. See: http:// creativecommons.org/licenses/by-nc/3.0/

\section{REFERENCES}

1. De Lau L, Breteler M. Epidemiology of Parkinson's disease. Lancet Neurol 2006;5:525-35.

2. Parkinson's Australia. Submission to: National Health and Hospitals Reform Commission: Parkinson's Australia; n.d. [cited 2013 February 15]. http://www.health.gov.au/internet/nhhrc/publishing.nsf/Content/ 143/\$FILE/143\%20Parkinson's\%20Australia\%20Submission.pdf

3. Horstink M, Tolosa E, Bonuccelli U, et al. Review of the therapeutic management of Parkinson's disease. Report of a joint task force of the European Federation of Neurological Societies and the Movement Disorder Society-European Section. Part I: early (uncomplicated) Parkinson's disease. Eur J Neurol 2006;13:1170-85.

4. A.D.A.M. Medical Encyclopedia. Parkinson's disease 2013 [cited 2012 June 20]. http://www.ncbi.nlm.nih.gov/pubmedhealth/ PMH0001762/

5. Van Nimwegen M, Speelman A, Overeem S, et al. Promotion of physical activity and fitness in sedentary patients with Parkinson's disease: randomised controlled trial. BMJ 2013;346:f576.

6. Ornish D, Brown S, Scherwitz L, et al. Can lifestyle changes reverse coronary heart disease? The lifestyle heart trial. Lancet 1990;336:129-33.

7. Ornish D, Weidner G, Fair W, et al. Intensive lifestyle changes may affect the progression of prostate cancer. $J$ Urol 2005;174:1065-9.

8. Ott M, Norris R, Bauer-Wu S. Mindfulness meditation for oncology patients: a discussion and critical review. Integr Cancer Ther 2005;5:98-108.

9. Grossman $\mathrm{P}$, Tiefenthaler-Gilmer U, Raysz A, et al. Mindfulness training as an intervention for fibromyalgia: evidence of postintervention and 3-year follow-up benefits in well-being. Psychother Psychosom 2007;76:226-33.

10. Pradhan E, Baumgarten M, Langenberg $P$, et al. Effect of mindfulness-based stress reduction in rheumatoid arthritis patients. Arthritis Rheum 2007;57:1134-42.

11. Morone N, Greco C, Weiner D. Mindfulness meditation for the treatment of chronic low back pain in older adults: a randomized controlled pilot study. Pain 2008;134:310-19.

12. Bohlmeijer E, Prenger R, Taal E, et al. The effects of mindfulness-based stress reduction therapy on mental health of adults with a chronic medical disease: a meta-analysis. J Psychosom Res 2010;68:539-44.

13. Kabat-Zinn J. Full catastrophe living —using the wisdom of your body and mind to face stress, pain, and illness. 3rd edn. New York: Bantam Dell, 2005.

14. Segal Z, Williams J, Teasdale J. Mindfulness-based cognitive therapy for depression:a new approach to preventing relapse. New York: Guliford Press, 2002.

15. Frattaroli J, Weidner G, Dnistrian A, et al. Clinical events in prostate cancer: results from two years of follow-up. J Urol 2008;72:1319-23.

16. Ornish D, Lin J, Daubenmier J, et al. Increased telomerase activity and comprehensive lifestyle changes: a pilot study. Lancet Oncol 2008;9:1124.

17. Hassed C. The ESSENCE of healthcare. Aust Fam Physician 2005;34:957-60.

18. Jelinek G, Hassed C. Managing multiple sclerosis in primary care: are we forgetting something? Qual Prim Care 2009;17:55-61.

19. Joyce A, Etty-Leal J, Zazryn T, et al. Exploring a mindfulness meditation program on mental health of upper primary children: a pilot study. Adv Sch Ment Health Promot 2010;3:16-24.

20. Hassed C, De Lisle S, Sullivan G, et al. Enhancing the health of medical students: outcomes of an integrated mindfulness and lifestyle program. Adv Health Sci Educ Theory Pract 2009;14:387-98.

21. Hassed C, Sierpina V, Kreitzer M. The health enhancement program at Monash University medical school. Explore 2008;4:394-7.

22. Hoehn M, Yahr M. Parkinsonism: onset, progression and mortality. Neurology 1967;17:427-42.

23. Michael J Fox Foundation. Clinical trials recruitment best practices manual. Michael J Fox Foundation, 2011 [cited 2012 October 8]. https://www.michaeljfox.org/research/trial.htm

24. Jenkinson C, Fitzpatrick R, Peto V, et al. The Parkinson's Disease Questionnaire (PDQ-39): development and validation of a Parkinson's disease summary index score. Age Ageing 1997;26:353-7.

25. Jenkinson C, Fitzpatrick R, Peto V, et al. The Parkinson's Disease Questionnaire: PDQ-39 User Manual (including PDQ-8 and the PDQ Summary Index). In: Evaluation of the Dutch version of the Parkinson's Disease Questionnaire 39 [Internet]. Oxford: Health Services Research Unit. 2nd edn. Parkinsonism and Related Disorders, 2008:24-7.

26. Lancaster G, Dodd S, Williamson P. Design and analysis of pilot studies: recommendations for good practice. J Eval Clin Pract 2010;10:307-12.

27. Vollestad J, Sivertsen B, Nielsen G. Mindfulness-based stress reduction for patients with anxiety disorders: evaluation in a randomized controlled trial. Behav Res Ther 2011;49:281-8. 
28. Shapiro S, Brown K, Thoresen C, et al. The moderation of mindfulness-based stress reduction effects by trait mindfulness: results from a randomized controlled trial. J Clin Psychol 67:267-77.

29. Schroevers $M$, Brandsma $R$. Is learning mindfulness associated with improved affect after mindfulness-based cognitive therapy? $\mathrm{Br} \mathrm{J}$ Psychol 2010;101(Pt 1):95-107.

30. Geoffrey C. Research Randomizer: Urbaniak and Scott Plous. Social Psychology Network; 2013 [cited 2012 June 14]. http://www. randomizer.org

31. Beller E, Gebski V, Keech A. Randomisation in clinical trials. Med J Aust 2002;177:565-7.

32. Australian Bureau of Statistics. National health survey: users' guideelectronic publication, 2007-08. Australian Bureau of Statistics, 2009 [cited 2012 June 2]. http://www.abs.gov.au/AUSSTATS/abs@.nsf/ allprimarymainfeatures/2851D0FD9C52AB56CA257ACC000E3DE1

33. Lovibond S, Lovibond P. Manual for the depression anxiety stress scales. 2nd edn. Sydney: Psychology Foundation, 1995.

34. Kenneth A, Wallston J. The validity of the multidimensional health locus of control scales. J Health Psychol 2005; 10:623-31.

35. Walach $\mathrm{H}$, Buchheld N, Buttenmuller V, et al. Measuring mindfulness-the Freiburg Mindfulness Inventory (FMI). Pers Individ Dif 2006;40:1543-55.

36. Vandenberg B; On behalf of the ESSENCE Research Team ESSENCE how to fill out the surveys: You Tube; 2012.

37. Hays R. The medical outcomes study (MOS) measures of patient adherence 2013 [cited 2012 April 4]. http://www.rand.org/content/ dam/rand/www/.../mos_adherence_survey.pdf
38. King D, Lashley R. A quantifiable alternative to double data entry. Control Clin Trials 2000;21:94-102.

39. Webb P, Bain C. Essential epidemiology: an introduction for students and health professionals. 2nd edn. New York: Cambridge University Press, 2011

40. Schensul S, Schensul J, LeCompte M. Essential ethnographic methods: observations, interviews and questionnaires. Walnut Creek: Alta Mira Press, 1999

41. Borkan J. Immersion/Crystallization. In: Crabtree B, Miller W. eds. Doing qualitative research. 2nd edn. Sage Publications, 1999:179-94.

42. Boeije H. A purposeful approach to the vonstant comparative method in the analysis of qualitative interviews. Qual Quantity 2002;36:391-409.

43. Schmidt S, Grossman P, Schwarzer B, et al. Treating fibromyalgia with mindfulness-based stress reduction: results from a 3-armed randomized controlled trial. Pain 2011;152:361-9.

44. Chiesa A, Calati R, Serretti A. Does mindfulness training improve cognitive abilities? A systematic review of neuropsychological findings. Clin Psychol Rev 2011;31:449-64.

45. Weber B, Jermann F, Gex-Fabry M, et al. Mindfulness-based cognitive therapy for bipolar disorder: a feasibility trial. Eur Psychiatry 2010;25:334-7.

46. Teixeira $\mathrm{E}$. The effect of mindfulness meditation on painful diabetic peripheral neuropathy in adults older than 50 years. Holist Nurs Pract 2010;24:277-83.

47. Emerson R, Fretz R, Shaw L. Writing ethnographic fieldnotes. Chicago: University of Chicago Press, 1995. 